\title{
Technical Note: Accuracy and repeatability of a new portable ultrasound pachymeter
}

\author{
A. Queirós ${ }^{1}$, J. M. González-Méijome ${ }^{1}$, P. Fernandes ${ }^{1}$, J. Jorge ${ }^{1}$, \\ J. B. Almeida ${ }^{1}$ and M. A. Parafita ${ }^{2}$ \\ ${ }^{1}$ Department of Physics (Optometry), School of Sciences, Campus de Gualtar, University of Minho, \\ 4710-057 Braga, Portugal, and ${ }^{2}$ Department of Surgery (Ophthalmology), School of Optics and \\ Optometry, University of Santiago de Compostela, Santiago de Compostela, Spain
}

\begin{abstract}
Purpose: To assess the accuracy and repeatability of central corneal thickness (CCT) measurements taken with a new portable ultrasound (US) pachymeter.

Methods: Central thickness measurements were taken with a portable pachymeter (SP-100 Handy; Tomey, Nagoya, Japan) and a conventional US pachymeter (Nidek UP-1000; Nidek Technologies, Gamagori, Japan) from 57 right corneas of 57 young adults (19 males, 38 females) aged 18-44 years (mean \pm S.D., $22.95 \pm 3.92$ ). Three repeated measures were obtained and then compared to obtain the repeatability of each instrument and the agreement between the pachymeters. The three readings taken with the portable pachymeter were compared against each other in order to evaluate intra-session repeatability and bias of each individual measurement with respect to the mean of three.

Results: Mean values of CCT were $537 \pm 35 \mu \mathrm{m}$ for conventional and $534 \pm 35 \mu \mathrm{m}$ for the new portable pachymeter. A high agreement was found between the two instruments (mean difference $=2.58 \mu \mathrm{m} ; 95 \% \mathrm{Cl} 1.41-3.75 \mu \mathrm{m}$ ) with only two eyes presenting differences larger than $\pm 8.6 \mu \mathrm{m}$ which represents $95 \% \mathrm{Cl}$ in the Bland-Altman plots which represents $1.6 \%$ of the mean CCT. The first reading taken showed the highest agreement with the mean value for the portable pachymeter.

Conclusions: The instrument tested in this study is able to take reliable measurements of corneal thickness even if a single reading is considered. Intra-session repeatability was very high, as was also the agreement between the average of three readings taken with the two US pachymeters.
\end{abstract}

Keywords: corneal thickness, pachymeter comparison, portable ultrasound pachymetry

\section{Introduction}

Corneal thickness (CT), as measured by pachymetry, is a sensitive indicator of corneal health and physiological performance. Despite the numerous new pachymetric techniques available, ultrasound (US) technology is one of the most commonly accepted in terms of accuracy, being the gold standard against which most of the new

Received: 30 January 2006

Revised form: 4 April 2006

Accepted: 30 April 2006

Correspondence and reprint requests to: Antonio Queirós Pereira.

Tel.: + 3512536043 33; Fax: + 351253678981 .

E-mail address: aqp@fisica.uminho.pt devices have been tested (Bovelle et al., 1999; Marsich and Bullimore, 2000; Iskander et al., 2001; Modis et al., 2001; Wirbelauer et al., 2002, 2004; González-Méijome et al., 2003; Lackner et al., 2005). A recent investigation involving two US pachymeters, confocal microscopy and slit scanning pachymetry, concluded that both US pachymeters gave the most consistent measurements of CT with standard deviations of the difference between two consecutive measurements as small as 6 and $7 \mu \mathrm{m}$ (McLaren et al., 2004).

Despite the need for contact with the cornea, US pachymetry is still at the forefront of the techniques used to obtain rapid, accurate and reproducible measurements of $\mathrm{CT}$ at a reasonable cost, when compared with other currently available devices. Portability of clinical instruments allows their use outside the clinical 
environment, making them excellent tools for screening and field data acquisition.

In the present study, we aimed to validate a new portable US pachymeter for the measurement of central corneal thickness (CCT). This is an important issue because of the potential usefulness of the device in clinical practice due to its relatively reduced cost and portability.

\section{Material and methods}

Fifty-seven right eyes from 57 patients (19 males, 38 females), with ages ranging from 18 to 44 years (mean \pm S.D., $22.95 \pm 3.92$ years), were selected to participate in this study. Slit-lamp examination and interview were carried out prior to data acquisition in order to ensure that none of the subjects exhibited corneal disease or corneal scarring, had been previously submitted to corneal refractive surgery, nor were taking any ocular or systemic medication. The research protocol followed the tenets of the Declaration of Helsinki and was reviewed and approved by the Scientific Committee of the School of Sciences of Minho University (Portugal). After explaining the nature of the experimental procedures, informed consent was obtained from each subject prior to data acquisition.

Corneal thickness was measured with a conventional US biometer/pachymeter, the Nidek UP-1000 (Nidek Technologies, Gamagori, Japan) and a new portable pachymeter, the SP-100 Handy pachymeter (Tomey, Nagoya, Japan). This new portable pachymeter operates at $20 \mathrm{MHz}$ measuring thicknesses in the range from 150 to $1200 \mu \mathrm{m}$ at calibration speeds ranging from 1400 to $2000 \mathrm{~m} \mathrm{~s}^{-1}$.

One drop of $1 \%$ tetracaine hydrochloride was instilled before pachymetric readings were taken by a trained clinician avoiding excessive compression of the tip probe against the cornea. Both pachymeters were calibrated prior to data acquisition at each measurement session: calibration was accepted when five measurements of the test block were taken with an accuracy of $\pm 1 \mu \mathrm{m}$. US speed was set at $1640 \mathrm{~m} \mathrm{~s}^{-1}$ for a vibration frequency of $20 \mathrm{MHz}$ in both instruments according to the values most commonly used in experiments involving human corneas and the recommendations of most of the manufacturers.

In order to avoid the potential effect of epithelial compression on consecutive measurements at the same location, the instruments were used in random order. To ensure the repeatability of the positioning of the pachymeter in subsequent measurements, a fixation panel was placed in front of the patient so that the probe tip contacted the cornea at the visual center. This system has been previously used successfully to obtain reproducible central and peripheral CT measurements with US pachymetry (Parafita et al., 1999, 2000;
González-Méijome et al., 2003). Three repeated measurements were taken consecutively with each instrument and averaged for subsequent comparisons.

As CT measurements taken by US pachymetry could be adversely affected by fluctuations in tissue hydration related to US speed through the cornea, contact lens users were excluded from the study. All measurements were recorded in the afternoon, between 16.00 and 20.00 hours, considered as the most stable part of the day for thickness fluctuations and, during this period, US speed through the cornea would be expected to agree with the calibration settings of the US probe (Du et al., 2003).

Data were analysed using the statistical package SPSS version 14.0 (SPSS Inc., Chicago, IL, USA). Correlations between central and peripheral measurements were assessed statistically as the mean of the differences compared with zero. The $95 \%$ limits of agreement $(\mathrm{LoA}=$ mean of the difference $\pm 1.96 \times$ S.D. of the differences) were also calculated (Bland and Altman, 1986). After normality and equality of variances were assessed, parametric tests were performed to assess the bias between the instruments. The hypothesis of zero bias was examined by Student's $t$-test. The level of significance was established at $\alpha=0.05$.

\section{Results}

Mean CCT was $537 \pm 35 \mu \mathrm{m}$ for the Nidek UP-1000 and $534 \pm 35 \mu \mathrm{m}$ for the Tomey SP-100 Handy pachymeter. The mean difference between the instruments was $2.58 \pm 4.39 \mu \mathrm{m}$ which was statistically different from zero $(t=4.44 ; p<0.001$; paired $t$-test $)$. However this value is unlikely to have any clinical significance. A plot of the differences between the measurements from the two instruments is presented in Figure 1, displaying a high agreement between the two instruments with only two eyes beyond the $95 \%$ limits of agreement. There is a

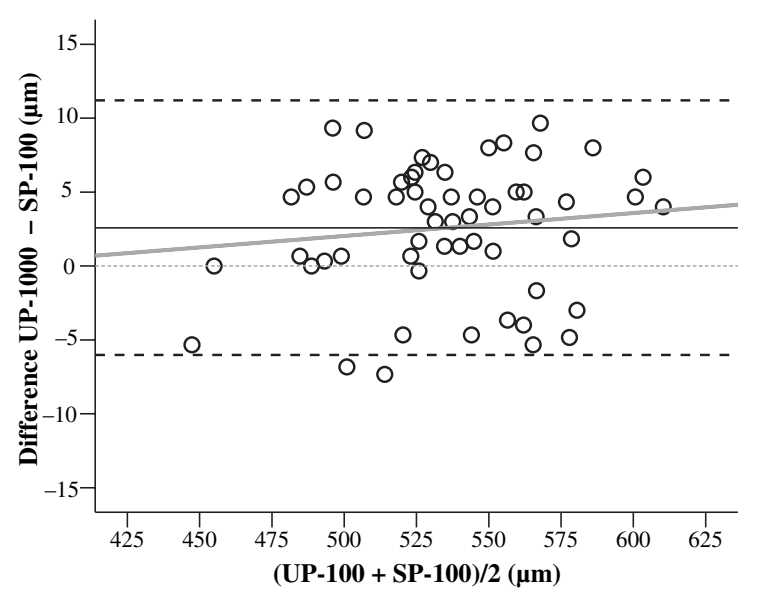

Figure 1. Plot of difference against mean central corneal thickness values obtained with the two pachymeters $(r=0.124 ; p=0.359)$. 
Table 1. Descriptive statistics of the three measurements taken within the same session at corneal centre with the Tomey SP-100, and the mean values obtained with the Nidek UP-1000. Units are microns $(\mu \mathrm{m})$

\begin{tabular}{lllll}
\hline & Minimum & Maximum & Mean & $\begin{array}{l}\text { Standard } \\
\text { deviation }\end{array}$ \\
\hline Tomey SP-100 & & & & \\
1st reading & 451 & 606 & 533 & 34 \\
2nd reading & 450 & 610 & 534 & 34 \\
3rd reading & 449 & 609 & 535 & 34 \\
$\quad$ Mean & 450 & 608 & 534 & 34 \\
Nidek UP-1000 & & & & \\
$\quad$ Mean & 445 & 612 & 537 & 35 \\
\hline
\end{tabular}

slight trend towards underestimation of higher CCT and overestimation of lower CCT by the portable Tomey SP-100 when compared with Nidek UP-1000. However this trend was not statistically significant $(r=0.124$; $p=0.359$ ). According to these data, we can show that CCT with the Tomey SP-100 Handy pachymeter can be obtained with a mean difference of $2.58 \pm 8.61 \mu \mathrm{m}$ when compared with a conventional (non-portable) pachymeter.

Table 1 presents mean, S.D., and maximum and minimum values within each one of the three readings taken with the Tomey SP-100 Handy pachymeter used to compute the mean value, and the comparison values for the Nidek UP-1000 described above. All the three measurements show a strong correlation with each other $(r>0.99 ; p<0.001)$. Only differences between the first and the third measurement were statistically different from zero $(t=-3.34 ; p=0.001)$. However the mean difference of $-1.42 \pm 3.21 \mu \mathrm{m}$ does not have any clinical relevance. Conversely, the stronger correlation $(r=0.996 ; p<0.001)$ and the least mean difference $(-0.63 \pm 3.94 \mu \mathrm{m})$ was found between second and third measurements $(t=-1.21 ; p=0.232)$.

Comparing each single reading taken with Tomey SP100 against the mean of three readings we found a closer agreement between average value and the first reading (mean difference \pm S.D. $=0.28 \pm 1.38 \mu \mathrm{m}$ ) followed by the second $(0.67 \pm 1.56 \mu \mathrm{m})$ and third one $(0.99 \pm 1.54 \mu \mathrm{m})$.

\section{Discussion}

The importance of CT is well known in many clinical situations, such as diagnosis of corneal ectatic conditions, investigation of corneal physiology, contact lens research or refractive surgery procedures. There are several optical methods to estimate the $\mathrm{CT}$, and a wide body of related literature is available regarding this subject (Parafita et al., 2002). Conversely, US pachymetry has been a standard for the estimation of CT for the last decades, and now that modern optically based pachymetric techniques are commercially available, US pachymetry is the reference against which they are all tested (Bovelle et al., 1999; Marsich and Bullimore, 2000; Iskander et al., 2001; Modis et al., 2001; Wirbelauer et al., 2002, 2004; González-Méijome et al., 2003; Lackner et al., 2005). Mean values and standard deviations found in the present study for the CCT of young healthy humans agree with the accepted values of CCT found in the literature on US pachymetry of normal corneas (Yaylali et al., 1997; Bovelle et al., 1999; Marsich and Bullimore, 2000; Doughty et al., 2002; González-Méijome et al., 2003; Lackner et al., 2005).

If we can assume a bias of $\pm 4 \mu \mathrm{m}$, one measurement will be enough with this instrument. These values are of the same order of magnitude or even slightly lower than those reported by other authors for two consecutive measurements with US pachymetry (Bovelle et al., 1999; McLaren et al., 2004) and supports the precision in pachymeter position among the repeated measurements. Gillis and Zeyen (2004) reported similar values for five repeated measurements with US pachymetry. Nevertheless, despite the fact that the first single reading has demonstrated the highest level of agreement with the average of the three, we recommend taking three repeated measurements to ensure accuracy. Moreover, in this situation, the last two readings demonstrated the highest intrasession agreement in the present study.

For the majority of the clinical applications, including refractive surgery, corneal health screening, contact lens effects on the corneal physiology or correction of intraocular pressure measurements, the new portable SP-100 US pachymeter obtains reliable measurements.

Obviously, when comparing instruments, the different principles on which they are based may well affect the level of agreement. This is the case for pachymetry when comparing US and optical methods, and all the new devices available in the marketplace are based on optical principles. Modified optical pachymetry has also shown a high degree of repeatability in measuring both total and epithelial thickness (Alharbi and Swarbrick, 2003; Perez et al., 2003; Alharbi et al., 2005). However its limited availability does not allow it to be considered as the 'gold standard' for most clinicians and investigators. Therefore, we suggest that future comparisons of central and peripheral CT measurements with different devices will include US pachymetry, as it is, in the hands of an experienced observer, the most reliable method for CT measurement.

In summary, the present study has evidenced the accuracy and repeatability of a new portable US pachymeter for the measurement of CCT in healthy human corneas. Portable instruments have several advantages for clinical and research applications, and the lower cost of these units is also an important advantage. 


\section{Acknowledgments and disclosure}

The authors wish to thank ISAZA for the loan of the Tomey SP-100 Handy Pachymeter. None of the authors has a commercial or financial interest in the instruments presented here.

\section{References}

Alharbi, A. and Swarbrick, H. A. (2003) The effects of overnight orthokeratology lens wear on corneal thickness. Invest. Ophthalmol. Vis. Sci. 44, 2518-2523.

Alharbi, A., La Hood, D. and Swarbrick, H. A. (2005) Overnight orthokeratology lens wear can inhibit the central stromal edema response. Invest. Ophthalmol. Vis. Sci. 46, 2334-2340.

Bland, J. M. and Altman, D. G. (1986) Statistical methods for assessing agreement between two methods of clinical measurement. Lancet 1, 307-310.

Bovelle, R., Kaufman, S. C., Thompson, H. W. and Hamano, H. (1999) Corneal thickness measurements with the Topcon SP-2000P specular microscope and an ultrasound pachymeter. Arch. Ophthalmol. 117, 868-870.

Doughty, M. J., Laiquzzaman, M., Muller, A., Oblak, E. and Button, N. F. (2002) Central corneal thickness in European (white) individuals, especially children and the elderly, and assessment of its possible importance in clinical measures of intra-ocular pressure. Ophthalmic Physiol. Opt. 22, 491-504.

Du, T. R., Vega, J. A., Fonn, D. and Simpson, T. (2003) Diurnal variation of corneal sensitivity and thickness. Cornea 22, 205-209.

Gillis, A. and Zeyen, T. (2004) Comparison of optical coherence reflectometry and ultrasound central corneal pachymetry. Bull. Soc. Belge. Ophtalmol., 292, 71-75.

González-Méijome, J. M., Cervino, A., Yebra-Pimentel, E. and Parafita, M. A. (2003) Central and peripheral corneal thickness measurement with Orbscan II and topographical ultrasound pachymetry. J. Cataract. Refract. Surg. 29, 125132.

Iskander, N. G., Anderson, P. E., Peters, N. T., Gimbel, H. V. and Ferensowicz, M. (2001) Accuracy of Orbscan pachymetry measurements and DHG ultrasound pachymetry in primary laser in situ keratomileusis and LASIK enhancement procedures. J. Cataract Refract. Surg. 27, 681-685.
Lackner, B., Schmidinger, G., Pieh, S., Funovics, M. A. and Skorpik, C. (2005) Repeatability and reproducibility of central corneal thickness measurement with Pentacam, Orbscan, and ultrasound. Optom. Vis. Sci. 82, 892-899.

Marsich, M. W. and Bullimore, M. A. (2000) The repeatability of corneal thickness measures. Cornea 19, 792-795.

McLaren, J. W., Nau, C. B., Erie, J. C. and Bourne, W. M. (2004) Corneal thickness measurement by confocal microscopy, ultrasound, and scanning slit methods. Am. J. Ophthalmol. 137, 1011-1020.

Modis, L., Jr, Langenbucher, A. and Seitz, B. (2001) Scanningslit and specular microscopic pachymetry in comparison with ultrasonic determination of corneal thickness. Cornea 20, 711-714.

Parafita, M., Yebra-Pimentel, E., Giraldez, M. J., GonzalezPerez, J., Perez-Martin, M. V. and Gonzalez-Meijome, J. (1999) Further information on the knowledge of topographical corneal thickness. Int. Contact Lens Clin. 26, 128-137.

Parafita, M. A., Gonzalez-Meijome, J. M., Diaz-Rey, J. A., Gonzalez-Perez, J. and Yebra-Pimentel, E. (2000) Corneal thickness mapping by topographical ultrasonic pachymetry. Int. Contact Lens Clin. 27, 12-21.

Parafita, M. A., Yebra-Pimentel, E., Giraldez, M. J., Gonzalez, J., Gonzalez-Meijome, J. M. and Cerviño, A. (2002) Optical methods for corneal thickness measurement: a review. In: Recent Research Developments in Optics (ed. S. G. Pandalai), Research Signpost, Trivandrum, Kerala, pp. 35-51.

Perez, J. G., Meijome, J. M., Jalbert, I., Sweeney, D. F. and Erickson, P. (2003) Corneal epithelial thinning profile induced by long-term wear of hydrogel lenses. Cornea 22, 304-307.

Wirbelauer, C., Scholz, C., Hoerauf, H., Pham, D. T., Laqua, H. and Birngruber, R. (2002) Noncontact corneal pachymetry with slit lamp-adapted optical coherence tomography. Am. J. Ophthalmol. 133, 444-450.

Wirbelauer, C., Aurich, H., Jaroszewski, J., Hartmann, C. and Pham, D. T. (2004) Experimental evaluation of online optical coherence pachymetry for corneal refractive surgery. Graefes. Arch. Clin. Exp. Ophthalmol. 242, 24-30.

Yaylali, V., Kaufman, S. C. and Thompson, H. W. (1997) Corneal thickness measurements with the Orbscan Topography System and ultrasonic pachymetry. J. Cataract Refract. Surg. 23, 1345-1350. 